Psychotherapeut 2022 $67: 28-33$

https://doi.org/10.1007/s00278-021-00552-z

Angenommen: 6. Oktober 2021

Online publiziert: 31. Oktober 2021

(c) Springer Medizin Verlag $\mathrm{GmbH}$, ein Teil von Springer Nature 2021

\section{In diesem Beitrag}

- Definitionen und Prävalenzen

- Psychische Entwicklung und der „Frühgeborenenverhaltensphänotyp"

- Frühgeburt und Übergang zur Elternschaft

Stress in der Schwangerschaft • Frühgeburt als Krise - Ängste, Depressivität und posttraumatische Belastung

- Frühgeburt und Intensivbehandlung: nachhaltige Erfahrung für das Kind? Toxischer Stress - Intensivmedizinische Umfeldbedingungen - Protektiv: Beispiel „Kängurupflege"

- Eltern-Kind-Interaktion und Beziehungsentwicklung

Das Frühgeborene als "schwieriger

Sozialpartner" • Belastete Eltern treffen

auf unreife Kinder

- Schlussfolgerung

\title{
Frühgeburt: Risiko für die psychische Gesundheit?
}

\author{
Wie elterliche Belastungen und frühkindliche \\ Entwicklungsbedingungen zusammenwirken
}

Carola Bindt

Klinik für Kinder- und Jugendpsychiatrie,-psychotherapie und -psychosomatik, Universitätsklinikum Hamburg-Eppendorf, Hamburg, Deutschland

\section{Zusammenfassung}

Jedes zwölfte Kind kommt vor der vollendeten 37. Schwangerschaftswoche zur Welt, und in den vergangenen Jahrzehnten haben sich die Überlebens- und Entwicklungschancen selbst sehr unreifer Kinder deutlich verbessert. Damit sind auch die psychischen Folgen einer Frühgeburt in den Fokus getreten. Die Manifestation eines psychopathologischen Profils mit Symptomen von Angst-, Aufmerksamkeits- und Autismus-Spektrum-Störungen betrifft insbesondere die Hochrisikogruppe von Kindern mit sehr niedrigem Geburtsgewicht und wird derzeit als „Frühgeborenenverhaltensphänotyp" diskutiert. Ausgehend vom aktuellen Forschungsstand zu den elterlichen psychischen Belastungen und frühkindlichen Entwicklungsbedingungen im Kontext einer Frühgeburt finden sich potenzielle Wirkfaktoren, die auch für die psychotherapeutische Praxis relevant sind.

\section{Schlüsselwörter}

Trauma $\cdot$ Neonatologische Behandlung $\cdot$ Neurologische Entwicklungsstörungen · Eltern-KindBeziehung · Psychotherapie

„Ich wurde viel zu früh geboren“. In der Psychotherapie löst diese Angabe oft Assoziationen zu einem fragilen, verlassenen, im Inkubator deprivierten Kind aus, für das Leben traumatisiert wie die Mutter, die ihr Kind nicht halten konnte und gleichfalls eine verfrühte Trennung verkraften musste. Inwieweit solche Vorstellungen die Realität abbilden, ist nicht leicht zu beantworten. Belegt ist, dass eine frühkindliche Stressbelastung vielfältige Gesundheitsprobleme vorhersagt. Im Kontext einer Frühgeburt wirken perinatale Risiken mit psychischen Folgen für Eltern und ihre Kinder ein.

\section{Definitionen und Prävalenzen}

Die Frühgeburt ist definiert als Geburt vor dervollendeten 37. Schwangerschaftswoche (SSW), wobei die Grenze der Überlebensfähigkeit in Westeuropa bei 23-24 SSW liegt (Smith et al. 2017). Gebräuchlich sind zudem Definitionen und Abkürzungen, die sich auf das Geburtsgewicht („birth weight", BW) als Indikator des Ausmaßes der Frühgeburtlichkeit beziehen: <2500 g ("low birth weight", LBW), <1500g (,very low birth weight", VLBW), <1000 g ("extremely low birth weight", ELBW). Auf der Grundlage dieser Definitionen lag der Anteil Frühgeborener in Deutschland 2017 bei 8,6\% aller Neugeborenen; knapp 20\% davon waren Mehrlingskinder (Institut für Qualitätssicherung und Transparenz im Gesundheitswesen 2018). Somit kam jedes zwölfte Kind zu früh zur Welt. Von allen Geborenen eines Jahrgangs sind etwas mehr als $1 \%$ Frühgeborene mit einem Geburtsgewicht $<1500 \mathrm{~g}$ und damit auf eine mehrmonatige, hochspezialisierte Behandlung angewiesen.

Trotz vielfältiger Anstrengungen, die Frühgeburtenrate zu senken, ist diese bis zum Ausbruch der durch die "coronavirus disease 2019" (COVID-19) ausgelösten Pandemie tendenziell angestiegen. Vermut- 
lich spielt das höhere Durchschnittsalter Schwangerer dabei ebenso eine Rolle wie die Zunahme von Maßnahmen der assistierten Reproduktion und komplizierten Mehrlingsschwangerschaften.

\section{Psychische Entwicklung und der "Frühgeborenenverhaltens- phänotyp"}

Die Frühgeburtlichkeit gilt als Risikofaktor für diverse psychopathologische Auffälligkeiten im Kindes- und Jugendalter. Auch das Risiko, im weiteren Lebensverlauf an einer Psychose, Depression oder bipolaren Störung zu erkranken, ist um das 1,3- bis 7-Fache der Norm erhöht (Nosarti et al. 2012). Dabei besteht eine inverse Relation zwischen dem Gestationsalter bei der Geburt und der Häufigkeit von psychischen Störungen (Woythaler 2019).

Die Profile psychischer Merkmale von Individuen mit sehr niedrigem Geburtsgewicht verweisen auf distinkte Muster von Symptomen dreier Störungsbilder (Aufmerksamkeitsdefizit- und Hyperaktivitätssyndrom [ADHS], Autismusspektrum, Angststörungen), die als „preterm behavioral phenotype" charakterisiert (Johnson und Marlow 2011) und aktuell vermehrt diskutiert werden (Fitzallen et al. 2020). Hierzu gehören ein ängstlich-zurückgenommener Beziehungsstil, Restriktionen in der sozialen Interaktion und im Verständnis für soziale Zusammenhänge. Frühgeborene weisen bei normaler Intelligenz zudem häufiger Defizite in den Aufmerksamkeitsleistungen und Exekutivfunktionen, der Sprachentwicklung sowie Teilleistungsstörungen auf. Alle Symptomkonstellationen haben vermutlich neurobiologische Ursachen, wobei Einflüsse der intrauterinen und auch frühen extrauterinen Entwicklungszeit und Beziehungsaspekte eingehen (Taylor 2020). Internalisierende Auffälligkeiten persistieren über die Lebensspanne.

Hinsichtlich der Besonderheiten in der sozialen Kognition und sozioemotionalen Anpassungsfähigkeit der empfindlichsten Gruppe unter den Frühgeborenen steht die Forschung noch am Anfang. Der Stand der Kenntnisse zu den Einflussfaktoren ist im Folgenden zusammengestellt.

\section{Frühgeburt und Übergang zur Elternschaft}

\section{Stress in der Schwangerschaft}

Obgleich die Ursachen für eine verkürzte Schwangerschaftsdauer vielschichtig sind, prägt das Bild vom seelischen "Schock", der eine Frühgeburt auslöst, die gängige Sicht. Die bisher erschlossenen Risiken sind komplex: Neben Faktoren wie uteroplazentaren Gefäßläsionen, Umwelt- und Genussgiften spielen genetische Prädispositionen und Gen-Umwelt-Interaktionen eine Rolle (Strauss et al.2018). Immunologische, über entzündlich wirkende Botenstoffe vermittelte Prozesse, die bei Infektionen, aber auch bei psychischer Stressbelastung zu beobachten sind und oft chronisch einwirken, können Frühgeburten begünstigen (Behura et al. 2019). Depressionen, Ängste, die Exposition gegenüber Rassismus und anderer "minority stress" sind als mitbedingend identifiziert worden. Stress in frühen Schwangerschaftsstadien scheint das Risiko sogar stärker zu erhöhen als solcher nach der 15. SSW. Hierzu fanden sich eindrucksvolle Ergebnisse in verschiedenen Studien, die sich mit den Auswirkungen der Terroranschläge in New York im September 2001 auf die Frühgeburtenrate befassten (Eskenazi et al. 2007). In jüngerer Vergangenheit stellte der "travel ban“ für Muslime in den USA eine Bedrohungslage dar. Die Analyse der US-Geburtenstatistiken der Jahre 2009-2018 ergab, dass dieses politisch begründete Einreiseverbot 2017 zu einem signifikanten Anstieg der Frühgeburten bei Frauen mit Ursprung in den betroffenen Ländern führte (Samari et al. 2020). Auch hier fanden sich Korrelationen mit einer Stressexposition in der Frühschwangerschaft.

Aufgrund der multifaktoriellen Genese lässt sich für den Einzelfall die Frage nach den Ursachen meist nicht beantworten. Dieser Umstand nährt elterliche Fantasien und Selbstvorwürfe, die in die Verarbeitung der Situation eingehen.

\section{Frühgeburt als Krise}

Eine Frühgeburt tritt nicht immer unerwartet ein, stürzt Eltern jedoch regelhaft in eine emotionale Krise. Die Begleitumstände können mit einer Todesbedrohung der
Mutter und auch des Kindes einhergehen. Dessen Unreife und neonatale Komplikationen stellen darüber hinaus die kindliche Unversehrtheit, oft langzeitig, infrage. Existenzielle Unsicherheiten und Zukunftsängste beeinträchtigen die Eltern nachhaltig, selbst wenn das Kind, wie es im Jargon der Neonatologie heißt, „es gut macht" und absehbar keine Schädigung davonträgt.

Neben objektivierbaren Risiken trägt das subjektive elterliche Erleben zur psychischen Belastung nach einer Frühgeburt bei; dieses ist vom Gefühl des Kontrollverlustes, von Schuldgefühlen, vom Leiden an der Trennung vom Kind und von der Unsicherheit in der Kontaktanbahnung unter Intensivstationsbedingungen geprägt (Flacking et al. 2006). Eine verkürzte Schwangerschaftsdauer und die Unmöglichkeit, für das Neugeborene verantwortlich Sorge tragen zu können, erschweren die emotionale Anpassung und die Übernahme der Elternrolle. Von verängstigten Eltern kaum zu bewältigen ist zudem die Anforderung, hochkomplexe medizinische Informationen aufnehmen und weitertragen zu müssen, was Scham und Insuffizienzgefühle intensivieren kann.

Reaktionen aus dem Umfeld, die von der Kontaktvermeidung bis zu intrusiven Nachfragen reichen, stören die Integration der Erlebnisse. War bei stark verkürzter Gestation die Schwangerschaft noch nicht sichtbar, kann das zu sozialen Irritationen führen. Die Bewältigung des Alltags während der stationären Behandlungszeit bringt viele Mühen mit sich, insbesondere wenn Geschwisterkinder zu versorgen und emotional aufzufangen sind.

Behandelnde orientieren sich bevorzugt am kindlichen Gesundheitszustand, um die emotionale Situation von Eltern abzuschätzen. Hierzu soll angemerkt sein, dass unverarbeitete Verlusterlebnisse, auch solche nach Fehlgeburten oder erfolglosen reproduktionsmedizinischen Behandlungen, durch eine Frühgeburt reaktiviert werden und unabhängig vom Gefährdungsgrad des Neugeborenen für das elterliche Befinden ausschlaggebend sein können (Ganseforth et al. 2002). Für Außenstehende sind solche nur auf Nachfrage zugänglich. Eltern mit vorbestehend vermehrter Ängstlichkeit, psychischen Erkrankungen und Traumatisierungen sind 
nach einer Frühgeburt besonders vulnerabel.

Ängste, Depressivität und posttraumatische Belastung

Schwangerschafts- und Geburtskomplikationen zählen zu den Risikofaktoren peripartaler psychischer Störungen. Zwei systematische Reviews zur mütterlichen Depressivität nach einer Frühgeburt (Vigod et al. 2010; de Paula Eduardo et al. 2019) verweisen auf eine mehrfach erhöhte Prävalenz im Vergleich zur Termingeburt. Mütter von reiferen Frühgeborenen sind ebenso betroffen wie solche, deren Kinder ein sehr niedriges Geburtsgewicht aufweisen. Prädiktiv für über 8 Monate hinaus persistierende mütterliche Symptome sind ELBW/VLBW, anhaltende Gesundheitsprobleme sowie Schlafstörungen des Kindes, Mehrlingsgeburten nach In-vitroFertilisation (IVF), mangelnde soziale Unterstützung und die „Lifetime“-Diagnose einer psychischen Erkrankung. Analog weisen Väter von ELBW-/VLBW-Kindern postpartal gleichfalls häufiger Symptome auf; diese korrelieren jedoch im Verlauf eher mit der mütterlichen Verfassung und allgemeinen Stressoren als mit der Gesundheit des Kindes. Erhöhte Depressionswerte bei beiden Eltern sowie beeinträchtigte Familienfunktionen sind auch noch 2 und 7 Jahre nach ELBW-/VLBWGeburt nachweisbar (Treyvaud 2014).

Postpartal finden sich zudem häufiger erhöhte elterliche Angstlevel, wobei sich Zusammenhänge mit dem kindlichen Geburtsgewicht sowie mit vorbestehender Ängstlichkeit, Stressbelastung unter der Geburt und niedrigem Selbstwert eruieren lassen (Helle et al. 2016). Im Fokus stehen die Angst vor dem Kind und dessen Pflege, vor der Verantwortung sowie gesundheits- und sicherheitsbezogene Ängste. Solche können mit beeinträchtigter Alltagsbewältigung einhergehen, sowie mit Schlafdeprivation und Erschöpfung wie bei der maternalen Trennungsangst, bei der die Mutter ihr Kind auch nachts ängstlich überwacht und keine Ruhe findet.

Unabhängig vom seltenen Vollbild einer posttraumatischen Belastungsstörung treten posttraumatische Symptome (PTS) nach Frühgeburt signifikant häufiger auf. Intrusionen (oft bezogen auf die Geburt),
Vermeidung (von kontextbezogenen Settings) sowie Übererregbarkeit finden sich bei 15-50\% aller Mütter und seltener auch bei Vätern, teilweise noch Monate nach der Klinikentlassung (Holditch-Davis et al. 2015; Helle et al. 2018). In Langzeitstudien persistieren Intrusionen und Übererregbarkeit bei solchen Frauen, die bereits kurz nach der Geburt deutlich erhöhte Stresswerte angeben (Kersting et al. 2004; Barthel et al. 2020).

\section{Frühgeburt und Intensivbehand- lung: nachhaltige Erfahrung für das Kind?}

Kommt ein Kind zu früh zur Welt, ist es in seinen Fähigkeiten zur Anpassung an die Umwelt unreif. Die Hirnentwicklung befindet sich im letzten Drittel der Schwangerschaft im Wachstumsspurt; Strukturaufbau und Synapsenbildung erfolgen extrauterin unter künstlich erschaffenen Bedingungen. In diesem Kontext ist relevant, ob dem Umfeld und der Behandlung in der Neonatologie selbst eine vulnerabilitätssteigernde Valenz zukommt.

\section{Toxischer Stress}

Ein Frühgeborenes gerät rasch in einen Zustand physiologischer Dysregulation, wenn es Pflegemaßnahmen, Schmerzen, Störungen seines Schlafes, Temperaturschwankungen und Hunger bewältigen muss. Die vielfältigen Anforderungen der Intensivtherapiezeit können als kumulative Stressexposition aufgefasst werden, der das Kind naturgemäß nicht entkommen kann.

In der Neonatologie ist das Neugeborene einer Vielzahl von Prozeduren ausgesetzt. Untersucher (Simons et al. 2003) zählten durchschnittlich 14 Eingriffe/Tag während der ersten beiden Therapiewochen, im Extrem bis zu 50. Solcherart Exposition und auch die häufige Unterbrechung des Schlafes werden derzeit als potenziell „toxischer Stress“ für das Kind und sein sich entwickelnde Gehirn diskutiert. Es ist anzunehmen, dass aversive Erfahrungen nicht spurlos bleiben, auch wenn die Langzeitfolgen noch kaum erfasst sind (Weber und Harrison 2019).

Unstrittig ist, dass frühkindliche Schmerzerfahrungen die Entwicklung des Zentral- nervensystems (ZNS) prägen. Vermittelt über neuronale und epigenetische Mechanismen scheinen diese zu den neuropsychologischen Auffälligkeiten beizutragen, die $25-50 \%$ aller Frühgeborenen mit Geburt vor der 32. SSW aufweisen. Je unreifer das Kind bei Geburt, desto größer das Risiko (Boggini et al. 2021). Schmerzen beeinträchtigen Architektur, Stoffwechsel und die Funktionalität verschiedener Hirnregionen. Zudem finden sich bei betroffenen Frühgeborenen noch im Alter von 18 Monaten deutlich erhöhte Spiegel von Stresshormonen. Eine prolongierte Stresshormonexposition wiederum kann neurobiologische Veränderungen bedingen, die Lern- und Gedächtnisfunktionen stören und mit persistierenden Verhaltensauffälligkeiten einhergehen (MooneyLeber und Brummelte 2020). Deshalb ist nicht auszuschließen, dass die psychopathologische Symptombelastung, die den Entwicklungsweg vieler frühgeborenen Kinder begleitet, in einer kumulativen Traumatisierung durch die neonatologische Intensivtherapie mitbegründet ist.

\section{Intensivmedizinische Umfeld- bedingungen}

Das Frühgeborene muss sich mit einem Umfeld auseinandersetzen, das von dem im Uterus stark abweicht. Neonatologien sind bemüht, die Lichtexposition anzupassen und Lärmbelästigung durch Respiratoren, schrille Monitorsignale etc. einzudämmen. Dennoch ist das Kind quantitativ und qualitativ anderen sensorischen Einflüssen ausgesetzt als im Schutzraum der Amnionflüssigkeit unter der mütterlichen Bauchdecke. Potenzieller Überstimulation steht die Unterstimulation der Propriozeption und des Gleichgewichtssinns gegenüber, die ein Ungeborenes üblicherweise erfährt, wenn es Körperbewegungen seiner Mutter mitmacht. Auch hört das Kind im Inkubator weniger Sprache als im Mutterleib. Es ist folglich invasiven Stimuli vermehrt ausgesetzt und von entwicklungsfördernden depriviert.

Wie sich solch atypische Konditionen langzeitig auswirken, ist weitgehend unklar. Einzelne Studien belegen, dass helles Licht negativ auf die zirkadiane Regulation und den Visus einwirkt (Watanabe et al. 2013). Nach Rekonfrontation mit 
der Geräuschkulisse der Intensivstation zeigen Kleinkinder in einigen Studien einen Anstieg der Herzfrequenz, der als Ausdruck einer belastenden Erinnerung gedeutet wird, in anderen nicht (Barreto et al. 2006).

Ausgefeilte Studiendesigns erbringen weitere Erkenntnisse: In der Neonatalperiode ist die Integration von basalen Stimulationsmerkmalen (Dauer, Rhythmus, Intensität von Reizen) ein erster kritischer Schritt bei der Reifung multisensorischer Verarbeitungswege. Ohne die sich allmählich entfaltende neuropsychologische Fähigkeit, den kontinuierlichen Strom an niedrigschwelligen Reizen aus der Umwelt zu kombinieren und zu bewerten, kann der Säugling nicht lernen, sein Erleben einzuordnen und emotionale Reaktionen darauf zu regulieren. Er ist dann hypothetisch einem Umfeld ausgesetzt, das unberechenbar und bedrohlich erscheint. Angesichts der Unreife des Gehirns und der oft unvorhersehbaren sensorischen Einflüsse, denen Frühgeborene wochen- oder monatelang ausgesetzt sind, stellt sich die Frage nach distinkten Entwicklungspfaden der Integration multisensorischer Erfahrungen bei diesen Kindern. Hierauf finden sich erste Hinweise in einer Untersuchung, die 61 früh- und 55 reifgeborene Kinder einschloss. Die Frühgeborenen weisen atypische Muster multisensorischer Integration, niedrige Reizschwellen für diverse Stimuli sowie internalisierende Verhaltensmerkmale wie ängstliches, rückzügiges Verhalten und hypersensitive Reaktionen auf, die als Anpassung an unzureichend integrierte postnatale Erfahrungen interpretiert werden können und mit dem typischen Verhaltensphänotyp korrespondieren (Maitre et al. 2020).

Zudem wurde erstmals erkundet, ob Kinder, die länger im Inkubator gepflegt werden, Ähnlichkeiten mit solchen zeigen, die im Säuglingsalter in personalarmen Institutionen versorgt und emotional depriviert werden. In diesem Sinn wurden neuropsychologische Profile von 299 sechsjährigen VLBW-Frühgeborenen, 101 rumänischen Waisen und 311 Reifgeborenen verglichen. Tatsächlich zeigen die Frühgeborenen und postinstitutionalisierten Kinder vergleichbare Auffälligkeiten in den Exekutivfunktionen und Selbstregula- tionsfähigkeiten, was die Vermutung nahelegt, dass es ähnlich gelagerte Risiken für die perinatale Hirnentwicklung gab. Hierzu zählen eine mütterliche perinatale Stressbelastung und ein verminderter Kontakt zu primären Bezugspersonen in den ersten Lebensmonaten (Reyes et al. 2019).

\section{Protektiv: Beispiel „Kängurupflege“}

Im Jahr 1978 evaluierten kolumbianische Kinderärzte eine Methode, die fragilen Neugeborenen dabei helfen sollte, ihren Wärmehaushalt zu regulieren und den Zugang zur Nahrungsquelle an der mütterlichen Brust zu sichern. Dies gelang, wenn Mütter ihre Kinder wie ein Känguru im kontinuierlichen Hautkontakt am Körper trugen. So entwickelten sich auch Frühgeborene, die sonst kaum überlebt hätten. Das „Kängu-ruhen“ wird mittlerweile routinemäßig in Perinatalzentren praktiziert, und die am elterlichen Körper gelagerten Kinder zeigen ein besseres Outcome. Positive Effekte sind bestätigt für Infektionsraten, die Regulation von Körpertemperatur und Schmerzreizen, Wachstumsdaten, die Stillentwicklung sowie die Qualität der Eltern-Kind-Beziehung; Ergebnisse zur motorischen und zur kognitiven Entwicklung sind uneindeutig (Conde-Agudelo und Díaz-Rossello 2016).

\section{Eltern-Kind-Interaktion und Beziehungsentwicklung}

\section{Das Frühgeborene als "schwieriger Sozialpartner"}

Seit den 1980er-Jahren werden videografierte Mutter-Kind-Interaktionen analysiert. Einvernehmlich werden Frühgeborene im Vergleich mit termingeborenen Babys als "schwierige Sozialpartner" charakterisiert. Sie suchen seltener und kürzer den Blickkontakt, zeigen weniger Mimik, Lächeln und Vokalisationen in der Beantwortung mütterlicher Zuwendung und imponieren in den ersten Lebensmonaten als weniger reagibel und dabei schneller überlastet. Ein oft abruptes Einschlafen im Sinne einer "protektiven Apathie“ bietet Reizschutz, bevor die Fähigkeit entwickelt ist, an externe Stimuli zu habituieren, was entwicklungspsychologisch als Reifezeichen gilt. Daneben zeigen sich Verhaltensweisen, die sich Eltern nicht intuitiv erschließen: Gähnen beispielsweise deutet eher Stress an als Müdigkeit, ebenso ein Strecken der Gliedmaßen und Finger sowie ein Schluckauf (Givrad et al. 2021).

Daneben scheinen sich Frühgeborene in grundlegenden Temperamentseigenschaften von Reifgeborenen zu unterscheiden. Es finden sich eine vermehrte motorische Aktivität, sensorische Empfindlichkeit und Intensität im Affekt, hingegen verminderte Aufmerksamkeits- und Ausdauerspannen in Abhängigkeit vom Grad der Frühgeburtlichkeit (Cassiano et al. 2020).

Frühgeborene Kinder haben folglich eingeschränkte Kapazitäten, die Rolle als Interaktionspartner ihrer Eltern zu übernehmen. Auch können sie diese weniger gut als reife Neugeborene nutzen, um Affekte zu modulieren und Verhaltenszustände zu organisieren. Mangelnde Stressregulationsfähigkeit und eine alterierte Verarbeitung sozialer Stimuli können den Aufbau befriedigender Eltern-KindBeziehungen im Sinne eines "child-toparent effect" erschweren.

\section{Belastete Eltern treffen auf unreife Kinder}

Bei Dyaden nach einer Frühgeburt imponiert einem Review zufolge in der Interaktion häufiger ein aktiver, direktiver und kontrollierender mütterlicher Stil. Unterschiede zur Termingeburt sind am deutlichsten in ersten Lebenshalbjahr der Kinder ausgeprägt und 2 Jahre lang nachweisbar; solche hinsichtlich der Bindungssicherheit finden sich nicht (Korja et al. 2012). Eine Metaanalyse, die Studien mit Kindern unterschiedlicher Reife, somatischer Belastung und Klinikbehandlungszeit einbezieht, findet keine signifikanten Differenzen in der mütterlichen Sensitivität nach Früh- und Reifgeburt. Die Analysen kontrollieren allerdings nicht für mütterliche psychische Belastungen und Störungen, da solche nicht miterfasst waren (Bilgin und Wolke 2015). Die Datenlage zur Mutter-Kind-Interaktion nach einer Frühgeburt ergibt somit kein einheitliches Bild; Interaktionsuntersuchungen mit Vätern sind rar. Vermutlich ist in diesem Zusammenhang eine dif- 
ferenziertere Betrachtung von elterlichen und kindlichen Wirkfaktoren notwendig, die über den Kennwert der Frühgeburt selbst hinausgeht.

Stress und Angst prägen bekanntlich elterliches Verhalten und die frühe Kindesentwicklung. Erhöhte mütterliche Angst in der Neonatalzeit ist ein unabhängiger Prädiktor für eine weniger sensitive Interaktion, ein schlechteres kognitives Outcome und mehr internalisierende Symptome bei 2-jährigen VLBW-Kindern, und die Ausprägung mütterlicher PTS 6 Monate nach der Frühgeburt korreliert negativ mit der Qualität der Interaktion nach 12 Monaten (Petit et al. 2016).

Indikatoren postpartaler mütterlicher Depression in der Interaktion, die sich vermehrt bei Mutter-Frühgeborenen-Dyaden finden, sagen kindliche psychische Auffälligkeiten im Schulalter sowie Defizite in der Emotionserkennung und in den Exekutivfunktionen vorher (Priel et al. 2020). Vermutlich sind Frühgeborene diesbezüglich sogar noch empfänglicher als reife Säuglinge. So manifestieren sich Effekte mütterlicher Depressivität auf basale Kortisolspiegel häufiger bei VLBW-Kindern und werden interpretiert als erhöhte Irritabilität gegenüber aversiver Stimulation, die sich in einer Dysregulation der neurohormonellen Stressachse ausprägt (Bugental et al. 2008).

Belastete Eltern frühgeborener Kinder zeigen sich offenbar vermehrt intrusiv, um ihre primär weniger responsiven Kinder im Kontakt zu engagieren. Dieser Stil der intensivierten, weniger feinfühligen Stimulation wird als "kompensatorisches Elternverhalten" beschrieben und birgt ein zusätzliches Risiko, da hieraus dauerhafte, sich gegenseitig verstärkende Fehlanpassungen erwachsen können. Die Kinder stellen sich beispielsweise auf einen kontrollierenden mütterlichen Interaktionsstil mit zwanghaft-angepasstem Beziehungsverhalten ein und sind im Kleinkindalter weniger gut entwickelt als Frühgeborene mit sensitiven Müttern. Zudem können psychische Belastungen der Eltern in den ersten postpartalen Monaten mit einer dauerhaft veränderten Wahrnehmungseinstellung in Bezug auf das Kind, Überprotektion und sogar der Zuschreibung von Psychopathologie einhergehen; selbst gesunde Frühgeborene werden häufiger als fragil und verhaltensauffällig wahrgenommen (Helle et al. 2019). Solche Attribuierungen wiederum sind mit einer vermehrten Inanspruchnahme medizinischer Dienste unabhängig von somatischen Risiken assoziiert (De Ocampo 2003).

\section{Schlussfolgerung}

Das einzigartige Zusammenspiel der Risiken einer Frühgeburt für die kindliche Hirnentwicklung, die elterliche psychische Gesundheit und die frühe Eltern-Kind-Beziehung bietet Erklärungsmodelle für die hohe Gefährdung Frühgeborener in Bezug auf die Ausbildung psychopathologischer Auffälligkeiten.

Aktuelle Forschungsergebnisse legen charakteristische Prozesse bei der Adaptation an die extrauterine Umwelt und der Verarbeitung von sozialen Stimuli bei Frühgeborenen nahe, die sich in der Stressregulation, sozialen Interaktion, Kognition und Verhaltensorganisation bis ins Erwachsenenalter ausprägen können. Zusätzlich relevant sind mütterliche Einflussfaktoren in der Schwangerschaft sowie elterliche psychische Belastungen und deren Effekte in der Eltern-Kind-Interaktion. Die emotionale Bewältigung der Situation seitens der Eltern wirkt darauf ein, wie sich das Entwicklungspotenzial des frühgeborenen Kindes entfalten kann. Gleichzeitig bildet diese, neben den neonatologischen Therapie- und Handling-Optionen, einen zentralen Ansatzpunkt für protektive Interventionen.

Risikofaktoren für die elterliche psychische Gesundheit im Kontext einer Frühgeburt sind bekannt und werden in vielen Perinatalzentren regelhaft erfasst, um gefährdete Eltern gezielt ansprechen und unterstützen zu können. Für die psychotherapeutische Praxis ist der Einbezug von Erkenntnissen zur Frühgeburt ein weiterer wünschenswerter Schritt in der Versorgung einer vulnerablen Klientel, die zahlenmäßig wächst.

\section{Fazit für die Praxis}

- Jedes zwölfte Kind wird zu früh geboren.

- Betroffene Eltern leiden peripartal vermehrt unter klinisch relevanten Ängsten, Depressivität und posttraumatischem Stress.
- Die Frühgeburtlichkeit gehört zu den häufigsten Risiken psychischer Störungen im Kindes- und Jugendalter; es besteht ein Zusammenhang zwischen dem Gestationsalter bei Geburt und der Symptomausprägung.

- Aktuell wird ein „Frühgeborenenverhaltensphänotyp" diskutiert, der durch Symptom-Cluster aus den Bereichen Aufmerksamkeits-, Angst- und AutismusSpektrum-Störungen charakterisiert ist und mit Auffälligkeiten einhergeht, die bis ins Erwachsenenalter persistieren.

- Die psychische Entwicklung Frühgeborener wird durch neurobiologische Faktoren, die Umfeldbedingungen der Intensivbehandlungszeit und ein alteriertes Interaktionsangebot geprägt.

Korrespondenzadresse

PD Dr. med. Carola Bindt

Klinik für Kinder- und Jugendpsychiatrie, -psychotherapie und -psychosomatik, Universitätsklinikum Hamburg-Eppendorf Martinistr. 52, W 35, 20246 Hamburg, Deutschland

bindt@uke.de

\section{Einhaltung ethischer Richtlinien}

Interessenkonflikt. C. Bindt gibt an, dass kein Interessenkonflikt besteht.

Für diesen Beitrag wurden von der Autorin keine Studien an Menschen oder Tieren durchgeführt. Für die aufgeführten Studien gelten die jeweils dort angegebenen ethischen Richtlinien.

\section{Literatur}

Barthel D, Göbel A, Barkmann C, Helle N, Bindt C (2020) Does birth-related trauma last? Prevalence and risk factors for posttraumatic stress in mothers and fathers of VLBW Preterm and term born children 5 years after birth. Front Psychiatry 11:575429

Barreto ED, Morris BH, Philbin MK et al. (2006) Do former preterm infants remember and respond to neonatal intensive care unit noise? Early Human Development 82:703-707

Behura SK, Dhakal P, Kelleher AM, Balboula A, Patterson A, Spencer TE (2019) The brain-placental axis: therapeutic and pharmacological relevancy to pregnancy. Pharmacol Res 149:104468

Bilgin A, Wolke D (2015) Maternal sensitivity in parenting preterm children: a meta-analysis. Pediatrics 136:e177-e193

Boggini T, Pozzoli S, Schiavolin P, Erario R, Mosca F, Brambilla P, Fumagalli M (2021) Cumulative procedural pain and brain development in very preterm infants: A systematic review of clinical and preclinical studies. Neurosci Biobehav Rev 123:320-336

Bugental DB, Beaulieu D, Schwartz A (2008) Hormonal sensitivity of preterm versus full-term infants to 
the effects of maternal depression. Infant Behav Dev 31:51-61

Cassiano RGM, Provenzi L, Linhares MBM, Gaspardo CM, Montirosso R (2020) Does preterm birth affect child temperament? A meta-analytic study. Infant Behav Dev 58:101417

Conde-Agudelo A, Díaz-Rossello JL (2016) Kangaroo mother care to reduce morbidity and mortality in low birthweight infants. Cochrane Database Syst Rev. https://doi.org/10.1002/14651858. CD002771.pub2

De Ocampo A, Maclas MM, Saylor CF, Katikaneni LD (2003) Caretaker perception of child vulnerability predicts behavior problems of NICU graduates. Child Psychiatry Hum Dev 34:83-96

De Paula EJAF, De Rezende MG, Menezes PR, DelBen CM (2019) Preterm birth as a risk factor for postpartum depression: a systematic review and meta-analysis. J Affect Disord 259:392-403

Eskenazi B, Marks AR, Catalano R, Bruckner T, Toniolo PG (2007) Low birthweight in New York city and upstate New York following the events of September 11th. Hum Reprod 22:3013-3020

Fitzallen GC, Taylor HG, Bora S (2020) What do we know about the preterm behavioral phenotype? A narrative review. Front Psychiatry 11:154

Flacking R, Ewald U, NyqvistKH, Starrin B (2006) Trustful bonds: a key to "becoming a mother" and to reciprocal breastfeeding. Stories of mothers of very preterm infants at a neonatal unit. Soc Sci Med 62:70-80

Ganseforth C, Kribs A, Von Gontard A, Kleffner G, Pillekamp F, Roth B, Sticker EJ, SchmidtDenter U (2002) Die Bedeutung biologischer und psychosozialer Einflussfaktoren für das Belastungs- und Bewältigungserleben bei Müttern Frühgeborener $<1500 \mathrm{~g}$ in den ersten Lebensmonaten des Kindes. Z Geburtshilfe Neonatol 206:228-235

Givrad S, Hartzell G, Scala M (2021) Promoting infant mental health in the neonatal intensive care unit (NICU): A review of nurturing factors and interventions for NICU infant-parent relationships. Early Hum Dev 154:105281

Helle N, Barkmann C, Ehrhardt S, Von Der Wense A, Nestoriuc Y, Bindt C (2016) Postpartum anxiety and adjustment disorders in parents of infants with very low birth weight: Cross-sectional results from a controlled multicentre cohort study. J Affect Disord 194:128-134

Helle N, Barkmann C, Ehrhardt S, Bindt C (2018) Postpartum posttraumatic and acute stress in mothers and fathers of infants with very low birth weight: Cross-sectional results from a controlled multicenter cohort study. J Affect Disord 235:467-473

Helle N, Barkmann C, Ehrhardt S, Wense AV, Nestoriuc Y, Bindt C (2019) Internalizing symptoms in very low birth weight preschoolers: symptom level and risk factors from four rating perspectives in a controlled multicenter study. J Affect Disord 246:74-81

Holditch-Davis D, Santos H, Levy J, White-Traut R, O'Shea TM, Geraldo V, David R (2015) Patterns of psychological distress in mothers of preterm infants. Infant Behav Dev 41:154-163

Institut für Qualitätssicherung und Transparenz im Gesundheitswesen (2018) Bundesauswertung zum Erfassungsjahr 2017 - Geburtshilfe Qualitätsindikatoren

Johnson S, Marlow N (2011) Preterm birth and childhood psychiatric disorders. Pediatr Res 69:11R-18R

\section{Preterm birth: a risk for mental health? Interaction between parental distress and early childhood conditions of development}

One in 12 children is born before 37 completed weeks of gestation and during the last decades survival rates and developmental outcomes of even very premature children have significantly improved. As a result, mental health sequelae of preterm birth are more frequently addressed. The manifestation of a distinct psychopathological profile with co-occurrence of symptoms associated with attention deficit/hyperactivity, autism spectrum and anxiety disorders has been recognized and discussed as "preterm behavioral phenotype", mainly affecting high-risk children with very low birth weights. Current research findings on parental psychological distress and early childhood developmental conditions after preterm birth indicate contextual factors that are of relevance, also for clinicians in psychotherapeutic practice.

\section{Keywords}

Trauma - Neonatal care $\cdot$ Neurodevelopmental disorders · Parent-child relations · Psychotherapy

Kersting A, Dorsch M, Wesselmann U, Ludorff $K$, Witthaut J, Ohrmann P, Hornig-Franz I, Klockenbusch W, Harms E, Arolt V (2004) Maternal posttraumatic stress response after the birth of a very low-birth-weight infant. J Psychosom Res $57: 473-476$

Korja R, Latva R, Lehtonen L (2012) The effects of preterm birth on mother-infant interaction and attachment during the infant's first two years. Acta Obstet Gynecol Scand 91:164-173

Maitre NL, Key AP, Slaughter JC, Yoder PJ, Neel ML, Richard C, Wallace MT, Murray MM (2020) Neonatal multisensory processing in preterm and term infants predicts sensory reactivity and internalizing tendencies in early childhood. Brain Topogr 33:586-599

Mooney-Leber SM, Brummelte S (2020) Neonatal pain and reduced maternal care alter adult behavior and hypothalamic-pituitary-adrenal axis reactivity in a sex-specific manner. Dev Psychobiol 62:631-643

Nosarti C, Reichenberg A, Murray RM, Cnattingius S, Lambe MP, Yin L, MacCabe J, Rifkin L, Hultman CM (2012) Preterm birth and psychiatric disorders in young adult life. Arch Gen Psychiatry 69:E1-8

Petit AC, Eutrope J, Thierry A, Bednarek N, Aupetit L, Saad S, Vulliez L, Sibertin-Blanc D, Nezelof S, Rolland AC (2016) Mother's emotional and posttraumatic reactions after a preterm birth: the mother-infant interaction is at stake 12 months after birth. PLoS ONE 11:e151091

Priel A, Zeev-Wolf M, Djalovski A, Feldman R (2020) Maternal depression impairs child emotion understanding and executive functions: the role of dysregulated maternal care across the first decade of life. Emotion 20:1042-1058

Reyes LM, Jaekel J, Kreppner J, Wolke D, Sonuga-Barke E (2019) A Comparison of the effects of preterm birth and institutional deprivation on child temperament. Dev Psychopathol 32:1524-1533

Samari G, Catalano R, Alcalá HE, Gemmill A (2020) The Muslim Ban and preterm birth: Analysis of U.S. vital statistics data from 2009 to 2018 . Soc Sci Med 265:113544

Simons SHP, Van Dijk M, Anand KS, Roofthooft D, Van Lingen RA, Tibboel D (2003) Do we still hurt newborn babies?: a prospective study of procedural pain and analgesia in neonates. Arch Pediatr Adolesc Med 157:1058-1064
Smith LK, Blondel B, Van Reempts P, Draper ES, Manktelow BN, Barros H, Cuttini M, Zeitlin J (2017) Variability in the management and outcomes of extremely preterm births across five European countries: a population-based cohort study. Arch Dis Child Fetal Neonatal Ed 102:F400-f408

3rd Strauss JF, Romero R, Gomez-Lopez N, HaymondThornburg H, Modi BP, Teves ME, Pearson LN, York TP, Schenkein HA (2018) Spontaneous preterm birth: advances toward the discovery of genetic predisposition. Am J Obstet Gynecol 218:294-314.e2

Taylor HG (2020) Neurodevelopmental origins of social competence in very preterm children. Semin Fetal Neonatal Med 25:101108

Treyvaud K (2014) Parent and family outcomes following very preterm or very low birth weight birth: a review. Semin Fetal Neonatal Med 19:131-135

Vigod SN, Villegas L, Dennis CL, Ross LE (2010) Prevalence and risk factors for postpartum depression among women with preterm and low-birth-weight infants: a systematic review. BJOG Int J Obstet Gynaecol 117:540-550

Watanabe S, Akiyama S, Hanita T, Li H, Nakagawa M, Kaneshi Y, Ohta H (2013) Designing artificial environments for preterm infants based on circadian studies on pregnant uterus. Front Endocrinol 4:113

Weber A, Harrison TM (2019) Reducing toxic stress in the neonatal intensive care unit to improve infant outcomes. Nurs Outlook 67:169-189

Woythaler M (2019) Neurodevelopmental outcomes of the late preterm infant. Semin Fetal Neonatal Med 24:54-59 